\title{
Ferdinand Oertel Kommunikationsmix Eine Perspektive \\ Podiumsdiskussion und Workshops auf dem Trendmonitor 2003
}

Auf dem MDG-Trendmonitor 2003 in Köln wurden die Untersuchungsbefunde der Allensbach-Studie in einem Podiumsgespräch unter dem Thema „Kommunikationsmix. Eine Perspektive" diskutiert und anschliessend in Arbeitsgruppen besprochen. Ferdinand Oertel hat die zentralen Aussagen der Diskussion sinngemäß zusammengefasst. Anschliessend folgen Kurzberichte über die Ergebnisse der Arbeitsgruppen von den Leitern der Gespräche.

\section{Podiumsdiskussion}

Teilnehmer: Mag. Wolfgang Bergmann, Standard, Wien; Dr. Klaus Driever, Weltbild-Verlag, Augsburg; Ulrich Fischer, Katholische Fernseharbeit, Frankfurt; Dr. Erich Jooß, St. Michaelsbund, München; Dr. Rüdiger Schulz, Institut für Demoskopie, Allensbach; Moderator: Adolf Theobald, München.

Theobald: Es bedarf einer kleinen Vorbemerkung: Kommunikationsmix ist nicht so sehr als Diskussion darüber gedacht, wie die einzelnen Medien sich untereinander verständigen und organisieren könnten, sondern eher darüber, mit welchen heutigen und zukünftigen Mitteln ihr Auftrag gestaltet werden kann. Mein Vorschlag wäre, dass jeder Podiumsteilnehmer kurz sagt, was er auf diesem Gebiet tut.

Driever: Für uns bei Weltbild lautet gerade vor dem Hintergrund auch der religiösen Kommunikation die große Frage letztlich: Wie komme ich an die Menschen heran? Wie erreiche ich sie, um sie tatsächlich mit meiner Botschaft auch erreichen zu können? Dabei nutzen wir auch durchaus die säkulare Kommunikation, um religiöse Themen zu transportieren. Ein Beispiel ist die Zeitschrift ,Leben und Erziehen' aus unserem Haus, eine Elternzeitschrift mit einer Auflage von etwa 140.000, 150.000 , die liegt ganz normal an den Kiosken aus und damit transportieren wir fast subkutan auch andere Themen als reine Erziehungsfragen. „Leben und Erziehen“ hatte z. B. ein großes Extra-Heft zum Thema ,Taufe' mit den Fragen, die junge Eltern heute stellen: Was ist diese Taufe überhaupt? Wie gehe ich damit um? Wir versuchen also, eine Großzahl von Menschen zu erreichen, um sie dann mit religiösem Basiswissen zu versorgen. Und erstaunlich oder nicht erstaunlich, dieses Sonderheft mit dem Thema ,Taufe' war eines der erfolgreichsten der letzten 
zwei Jahre. Die Bedürfnisse sind da, aber man muss erst einmal an die Menschen herankommen.

Theobald: Herr Bergmann, Sie kommen aus Österreich und waren in Wien auf dem Gebiet kirchlicher Medien sehr erfolgreich. Um was ging es da?

Bergmann: Ich war von 1996 bis 1999 Direktor für Öffentlichkeitsarbeit und Kommunikation der Erzdiözese Wien, wo wir damals konzentriert das Projekt einer integrierten Gesamtkommunikation der Erzdiözese verfolgt haben. Dazu gehörte zunächst die Einführung eines profan gesagt - Mitgliedermagazins, das heißt eines Mediums, das damals zehnmal jährlich in alle Haushalte geliefert wurde, in denen zumindest ein Katholik gelebt hat, Gesamtauflage 700.000 Stück. Dieses Projekt ist auch über österreichische Grenzen hinaus diskutiert worden. Ein Zweites, das ich für ebenso wichtig halte, was aber viel weniger diskutiert wurde, war die Etablierung eines eigenen Mitarbeitermagazins in der Auflage von ca. 20.000 Stück für alle, die in irgendeiner Form zum Funktionärskreis gehören, vom Religionslehrer bis zum Pfarrgemeinderat, auch im monatlichen Direktversand. In die integrierte Medienkommunikation waren gleichfalls die Öffentlichkeitsarbeit und die Pressestelle integriert. Gleichzeitig erfolgte der Start des Privatradios ,Radio Stephansdom', dessen Gründungsgeschäftsführer ich war. Das war kein Radio im Sinne dessen, was man unter kirchlichem Radio versteht, sondern vom Formatradio her ein Klassikradio mit sehr wohldosierten kirchlichen Inhalten. In diese Gesamtkonzeption war schliesslich auch der Bereich des Dom-Verlages mit der Kirchenzeitung einbezogen, die damals einem gründlichen Relaunch und einer Neupositionierung unterzogen worden ist. Das alles sollte in einem Feldversuch ausprobiert werden und ist empirisch begleitet worden, und als wichtigstes Ergebnis kann abgeleitet werden, dass es keine Entweder-Oder-Frage gibt etwa zwischen der Kaufzeitung oder der Gratiszeitung, sondern nur die Frage des Sowohl-als-auch.

Theobald: Herr Fischer, Sie mixen auch ganz schön im Kommunikationsmix mit.

Fischer: Einerseits bin ich in Frankfurt im Büro der Katholischen Fernseharbeit schwerpunktmäßig für die Kirchenprogramme bei Pro7 und SAT1 tätig, und andererseits arbeiten in diesem Büro auch der Beauftragte für das ZDF und der für RTL. Ich habe zwei 50\%-Stellen, und mit denen versorgen wir für das Bistum Limburg die öffentlich-rechtlichen Sender und die privaten Sender in Hessen mit Kirchenprogrammen. Ich möchte ein Beispiel für einen funktionierenden Medienmix geben: Wir haben in Frankfurt den Kapuzinerpater Bruder Paulus, der kommentiert täglich im Internet die Bild-Zeitung und hat dort eine sehr hohe Quote 
von Menschen, die sich das anschauen oder sich per E-mail zukommen lassen. Daneben moderiert er auf SAT1 eine kleine Sendung, hat auf N24 eine eigene Talkshow, ist viel gefragt in sämtlichen anderen Talkshows, und kürzlich war ich mit ihm in Hamburg beim Chefredakteur der BildZeitung. Dort haben wir erreicht, dass er seine Bild-Zeitungs-Kommentierung jetzt täglich direkt mit der Bild-Zeitung verlinken kann. Das heißt: Bild-online gibt täglich an, wie man seine Seite kommt, und er schreibt jetzt auch in Bild immer eine Kolumne zum Thema ,Lebenshilfe'. Dieses kleine Beispiel zeigt, wie ich versuche, Kirchenprogramme für die Nebenbeihörer zu machen. Es ist ganz wichtig, dass wir neben den kirchlichen Special-Interest-Programmen und Redaktionen, die sicher notwendig sein mögen, auch im Sinne der Mehr-und-Mehr-Regel, Menschen dafür interessieren, vor allem aus dieser berühmten Zielgruppe bis 49 , irgendwann einmal auch in die Kernzielgruppe hineinzuwachsen.

Theobald: Dr. Jooß, kann ich Sie als einen Mix-Manager bezeichnen?

Jooß: Ich habe nichts gegen das Wort Manager. Meine Erfahrungen im Sankt Michaelsbund begannen als Assistent. Damals hatte der Sankt Michaelsbund die Münchner Katholische Kirchenzeitung und war gleichzeitig der Büchereiverband in Bayern. Gelernt habe ich sehr früh, dass es einen Kernbereich von Kirche gibt, der möglichst effektiv bedient werden muss. Wir haben damals begonnen, die katholischen öffentlichen Büchereien in pfarrlicher Trägerschaft umzuwandeln in Gemeinde- und Stadtbüchereien mit Trägerschaft der Kommune, also Einbezug der säkularen Strukturen. Dabei habe ich gelernt, dass der Öffnungsbereich ein harter Bereich und die säkulare Welt eine durchaus anspruchsvolle Welt ist, dass wir aber damit Schwellenhemmungen in hohem Maße überwinden können. Was den Medien-Mix betrifft: In den letzten Jahren hat unser Haus eine Abteilung Mediendienste aufgebaut. Diese Abteilung bedient Privatsender in Bayern mit kirchlichen Beiträgen sowohl im Radio- wie im Fernsehbereich und hat eine Internet-Redaktion, die inzwischen auch Dienstleister geworden ist. Für mich ist das Wort Dienstleister ein zentraler Begriff. Wir haben darüber hinaus Buchhandelsaktivitäten verstärkt, und das alles unter dem Gesichtspunkt des Vertriebes und der Auffächerung und Differenzierung des Vertriebes von kirchlichem Inhalt. Dabei haben wir in Bayern gute Erfahrungen auf der Kooperationsebene mit dem Evangelischen Presseverband gemacht. Es hat uns immer geholfen, wenn wir gemeinsam aufgetreten sind. Gestern ist ein Schlüsselsatz genannt worden: „Geschlossenheit überwinden und dadurch Anschlussfähigkeit gewinnen.“ Das ist für mich der zentrale Punkt. Wie können wir aus unseren eigenen Mauern wieder hinausgehen ins säkulare Umfeld und wie können wir Anschluss gewinnen an die Medienentwicklungen? Ein Stichwort: In Bayern ist in den letzten Jahren 
eine Jugendsenderkette entstanden mit Namen ,Galaxy und es gab enorme Berührungsängste im kirchlichen Bereich, hinaus in die Jugendsender zu gehen. Wir haben es uns gemeinsam mit dem Evangelischen Presseverband zugetraut und machen sehr gute Erfahrungen damit. Ich glaube, man muss sich also wirklich der Öffentlichkeit aussetzen. Ich bin aber auch der Meinung, dass wir wirtschaftlich denken müssen und Refinanzierung brauchen. Wir haben als Dienstleister durch Verhandlungen auch im Internet-Bereich eine wachsende Refinanzierungsquote erreicht, die für mich deshalb wichtig ist, weil wir so unabhängig agieren können. Letzter Gesichtspunkt: Die Oberflächenstruktur der Angebote ist durch die Allensbach-Untersuchung sehr genau beschrieben. Schwierig wird es immer dann, wenn es in die Struktur darunter geht, Stichwort ,Pfarrbrief'. Natürlich respektiere ich und bin froh darüber, dass Pfarrbriefe so gut angenommen werden. Aber man muss dann auch die Pfarrbriefe selbst inhaltlich analysieren, was sie denn beitragen für Kirche in der Gesellschaft in der Welt und wie sie daherkommen. Dann schaut das Bild plötzlich etwas anders aus.

Zwischenfrage aus dem Publikum: Im Printbereich, aber auch im elektronischen Bereich, vor allem im Internet wird der Markt immer mehr von kostenlosen Produkten überschwemmt, die nicht unter Marktbedingungen hergestellt werden. Verändert sich dadurch die Bereitschaft, für ein Medium zu zahlen und Abonnements einzugehen?

Driever: Im Internet kann durchaus Geld verdient werden, bei Weltbild haben wir im letzten Jahr über das Internet Umsätze in einer Größenordnung von etwa 70 Millionen Euro gemacht und keine Verluste geschrieben. Und auch ,amazon' hat in Deutschland 350 Millionen Euro Umsatz gemacht. Am Anfang sind sicherlich viele Informationen kostenlos ins Internet gegeben worden, mittlerweile rudern einige zurück, manche versuchen es anders anzupacken. Die Informationen, die im Internet für die breite Bevölkerung da sind, werden auch kostenlos bleiben. Aber es gibt auch elektronische Inhalte, die durchaus bezahlt werden, etwa im Fachbereich, zum Beispiel macht Elsa aus Holland, eine der größten Fachbereichsgruppen im Buchbereich, Umsätze mit elektronischen Inhalten in der Größenordnung von etwa 1,5 Milliarden Euro. Sobald Informationen wirklich hoch spezifisch sind, wird dafür bezahlt.

Bergmann: Zwei Erfahrungen zur Frage der kostenlosen Produkte und den Marktbedingungen. Bei unserer Tageszeitung, dem Standard in Wien, ist es so, dass wir steigende Abonnements haben, obwohl wir den gesamten Kontext gratis im Internet anbieten. Und gleichzeitig gewinnen wir über das Internet einen erheblichen Nachwuchs unserer Abonnenten. Also kein Verdrängungswettbewerb, sondern ein Sowohl als auch. Und die Erfahrung in der Erzdiözese Wien war gleichfalls, dass Gratisangebo- 
te nicht zur Abbestellung der Kirchenzeitung geführt haben. Da ist zwar oft die innerkirchliche Kontroverse vor allem zwischen Öffentlichkeitsarbeitern und Verlagsverantwortlichen, die sagen: „Na ja, ihr habt es ja leicht, ihr dürft das Geld ausgeben und wir müssen nach Marktbedingungen arbeiten und auch noch Geld verdienen. Das ist unfair." Die Gesamtkommunikation einer Diözese oder der Kirche insgesamt ist jedoch nicht daran zu messen, ob sie unter Marktbedingungen fair oder unfair agiert hat, sondern wie erfolgreich und wie wirksam sie gewesen ist und welcher Mitteleinsatz welchen Ergebnissen gegenübersteht. Die Kirche kann einen ihrer Grundaufträge, nämlich den der Verkündigung, nicht darauf abstellen: Verkündigung erfolgt, so lange wir damit Geld verdienen. Ich möchte die etwas belasteten Begriffe der Missionierung oder der Verkündigung dadurch ersetzen, dass die Kirche so etwas wie eine Bringschuld zu den Menschen hat, umso mehr zu denen, die schon einmal dafür zahlen, dass sie dieser Gemeinschaft angehören. Diese Bringschuld gilt es zu erfüllen, und wenn ich es ein bisschen zugespitzt formulieren darf: Paulus hat nicht so viele Briefe geschrieben, weil sie abonniert worden sind, sondern weil er der Meinung war, dass er etwas zu sagen hat.

Schulz: Ich habe im vergangenen Jahr eine große Studie für den Bundesverband der Deutschen Zeitungsverleger zu den Online-Angeboten von Tageszeitungen gemacht. Daraus war klar zu erkennen, dass die Gratismentalität schwierig zu beseitigen ist. $74 \%$ der User von OnlineAngeboten von Tageszeitungen sind nicht bereit, dafür Geld aufzuwenden, weil darin für sie zu wenig geldwerter Nutzen liegt. Wenn überhaupt, dann zahlt man für die Nutzung einer Archivfunktion, für E-Ticketing, also Zusatznutzen. Insofern ist es richtig: komplementäre Gratisnutzung, kein Verdrängungswettbeweb, nur an den Rändern. Wer ohnehin dem Printprodukt ferner steht, kann sich zufrieden geben mit dem Wenigen, was er im Online-Angebot von Tageszeitungen bisher bekommt. Ich glaube allerdings, dass auch die Auseinandersetzung um die Free Papers, die kostenlosen Tageszeitungen, noch nicht ausgestanden ist. So lange irgendwo Nutzwert kostenlos angeboten wird, wird man die Leute kaum dazu bringen, etwas dafür zu bezahlen.

Theobald: Ich glaube, Online ist kein Ersatz für Print. Das kann gar nicht sein, es ist ein ganz anderer Markt. Dazu Dr. Jooß.

Jooß: Die Frage nach kostenlosen Produkten, die nicht zu Marktbedingungen produziert werden, muss differenziert gesehen werden. Es gibt Produkte, für die wir einen Preis verlangen müssen, und zwar einen marktgerechten Preis, Stichwort Buch. Es gibt Produkte, die die Kirche im Sinne der Bringschuld anbieten muss, ich warne aber davor, einfach zu sagen, die werden nicht zu Marktbedingungen hergestellt. Ein Teil der 
Marktbedingungen ist selbstverständlicher Bestandteil der Produktion, nämlich die Frage: Wer hört sich das an? Wer nutzt es im Online-Bereich? Dafür gibt es verlässliche Zahlen, die auch Maßstab unseres Handelns sind und sein müssen. Für jeden Sender ist es eine Marktzahl, wenn er bei den Umfragen ermittelt, wie häufig das Verkündigungswort eingeschaltet wird oder ob es ein Ausschaltfaktor ist. Hier müssen wir unter Marktbedingungen produzieren, weil wir für die Sender nicht an den Bedürfnissen ihres Publikum vorbeiproduzieren dürfen. Also, Gratismentalität, das würde ich als Wort in diesem Kontext eher streichen, sie ist vielleicht eine Provokation im Online-Bereich. Für eine Kirchenzeitung müssen wir Geld verlangen, aber jede Kirchenzeitung tut gut daran, im Online-Bereich mit erweiterten Angeboten vertreten zu sein, die auch wieder in die Zeitung selbst zurückführen. Dann sind wir bei einem Mix, der sinnvoll ist und der sich gegenseitig befruchten kann. Natürlich darf auch ein Verkündigungswort im Privatsender seinen Preis haben. Von unseren landesweiten Sendern verlangen wir eine anteilige Kostenerstattung, sodass wir auf eine Refinanzierung von fast der Hälfte unseres Budgets kommen.

Theobald: Die Frage der Finanzierung ist eines, die andere Frage richtet sich nach dem Erfolg. Ist der Erfolg beim Internet messbar?

Schulz: Es gibt die Möglichkeiten, über die Page Impressions die User zu lokalisieren. Die Wirkung, die damit erzielt wird, ist damit natürlich noch nicht belegt und das bedarf nun wieder intensiven Nachhakens, insbesondere durch das Instrument der Panel-Analyse, wenn man die Leute über längere Zeiträume begleitet. Die Internet-Angebote im kirchlichen Bereich werden von der Mehrheit als sinnvoll und hilfreich empfunden, aber ob sie etwas bewirken, ist eine andere Frage.

Driever: Die Grundfrage ist tatsächlich: Erreichen wir die Menschen, gehen wir auch dorthin, wo die Menschen tatsächlich sind oder bleiben wir in unserer Abgeschlossenheit? Ich finde es gut, wenn Bistümer, Kirchenzeitungen und andere Institutionen eine eigene gute Internet-Website haben und auch untereinander vernetzt sind. Aber ich denke, das alleine wird nicht ausreichen, sondern man muss auch dorthin gehen, wo die Menschen sich dann im Internet wirklich treffen. Warum macht man nicht einen Begriff wie ,Internet-Mission' populär? Und schicken die Leute, ähnlich wie jetzt Bruder Paulus, dorthin, wo sich die Menschen tatsächlich treffen. Also zum Beispiel auf eine T-online. Eine T-online alleine hat 10 Millionen Benutzer und diese Menschen kommunizieren untereinander. Warum sind wir dort nicht präsent? Dorthin zu gehen, wo die Menschen sind und dann auch letztlich zu sagen, was wir zu sagen haben. Das Wichtigste ist, nicht im Geschlossenen zu bleiben, sondern auf die Leute zuzugehen und sie tatsächlich zu erreichen. Das ist 
mühsam. Man kann natürlich nicht messen, was es wirklich bewirkt hat, aber wenn man den Kontakt zu den Leuten schon gar nicht herstellt, kann man gar nichts bewirken.

Fischer: Wir haben im Internet eine gigantische Zahl an kirchlichem Inhalt von Institutionen, Diözesen, Verbänden, Serviceneinrichtungen. Ich glaube, unser Problem ist eher, dass es nicht vernetzt ist. Die Situation für ein großes Portal ist die, dass wir unter ,katholisch.de' bis vor kurzem einen halben Redakteur hatten, im Moment haben wir gar keinen, irgendwann kriegen wir mal wieder einen. Aber das ist gar nichts, wenn man vergleicht, was andere an Personal haben, um Internet-Seiten zu betreiben, zum Beispiel das „Handelsblatt“ mit 20 Leuten in der Internet-Redaktion. Wir sollten schon ein paar Leute mehr haben, mit denen wir zumindest ein kleines Internet-Portal machen könnten.

Theobald: Ist es auch eine Frage der Ausbildung derjenigen, die das machen? Sind vielleicht die Journalisten nicht richtig ausgebildet, um so etwas zu machen?

Driever: Das war vielleicht vor vier, fünf Jahren. Mittlerweile ist es so, dass es genug Angebote gibt. Das ifp in München wird bestätigen, dass die Journalisten und Medienmanager, die heute ausgebildet werden, wirklich multimedial denken und den Kommunkationsmix absolut verinnerlicht haben. Die zentrale Frage lautet: Wofür bildet das Institut aus, zum Beispiel auch um diese Geschlossenheit zu überwinden und katholische Stimmen auch tatsächlich woanders hören zu lassen. Das gilt nicht nur für das Internet, sondern auch für Zeitschriften, Zeitungen, genauso auch für den ganzen Bereich des Buches, worïber man seltener spricht als über das Internet und andere Medien. Auch für das Buch ist es sehr wichtig, dass es im Sortiment gut platziert ist.

$J o o ß$ : Für alle diese Medienbereiche gilt der gleiche Grundsatz der absoluten Professionalität. Ich habe es als die Schlüsselerkenntnis aus der neuen Untersuchung empfunden, dass wir hier deutlich verbesserte Noten kriegen. Das gilt für das Buch, auch das religiöse Buch. Das gilt, und das war für mich eine der positiven Überraschungen, für die Kirchenzeitungen. Das muss man auch in dem momentanen Klima des Infragestellens der Kirchenpresse einmal sehr deutlich sagen, dass sie gewonnen hat, was ihre eigenen Leistungen, ihr journalistisches Profil, ihre Inhalte betrifft. Das ist nicht ganz selbstverständlich, da drückt sich aus, dass in den Häusern wirklich mit den Ergebnissen der letzten Untersuchung gearbeitet wurde. Dass wir keine Leser gewonnen haben, ist eine andere Sache, hier sind auch Imagebildungen noch sehr hartnäckig und heftig im katholischen Milieu zu Hause. Aber auch da ist Profesionalität gefragt.

Bergmann: Ich möchte ebenfalls eine Lanze für die Professionalität 
brechen. Insbesondere, wenn es darum geht, auch neue Dinge zu entwickeln und auf eine neue Zielgruppe hin. Das wird selten aus bereits bestehenden Medien heraus geschehen können. Man kann nicht einen Internet-Auftritt aus einer Kirchenzeitungsredaktion heraus entwickeln oder auch nicht eine Mitgliederzeitung aus einer bestehenden Kirchenzeitungsredaktion heraus. Eines der Erfolgsrezepte unseres InternetAuftritts beim Standard war im Unterschied zu anderen Printmedien in Österreich sicher, dass sich eine eigene Mannschaft nur darum gekümmert hat. Umgekehrt muss es dann aber auch so sein: Wenn wir verschiedene Medien haben, muss die Vernetzung untereinander und die Kooperation untereinander und das Zuliefern zueinander funktionieren. Wir haben immer dieselben Geschichten, aber sie sind zielgruppenspezifisch völlig verschieden aufzubereiten. Und zwar mit Profis. Hierbei sind wir oft in einer schwierigen Situation, weil wir nicht fragen, ob diese Profis Menschen sind, die Katholiken sind, ausgetreten sind oder was auch immer. Wir dürfen, wenn wir uns Profis bedienen, sie vielleicht sogar von extern zukaufen, nicht Maßstäbe anlegen, die im übrigen die Hauptamtlichen und Berufenen in der Kirche auch nicht immer erfüllen können.

Zwischenfrage aus dem Publikum von Frau Dr. Steppacher: Mir stellt sich nach meiner kurzen Erfahrung im Bereich katholischer Medien so dar, dass im Grunde an sehr vielen heterogenen Orten ein ganz hohes Wissen herrscht, aber dieses Wissen einfach intern zu schlecht vernetzt wird. Ich wünschte mir, dass die Kirche versuchen würde, gute Wissensstände weiterzuleiten, sodass nicht jeder immer wieder bei Null anfangen muss. Dazu gehört auch, dass man Organisationsstrukturen überwindet. Ich habe oft den Eindruck, dass stark von bestehenden Strukturen gedacht wird und nicht von den Nutzern oder Lesern oder Käufern. Da stellt sich für mich konkret die Frage: Ist das Bistum als eine Bezugsgröße überhaupt relevant für den Nutzer, für den Leser? Das ist die eine Geschichte und die zweite Geschichte: Beim Pfarrbrief teile ich die Auffassung von Dr.Jooß, dass es da unterschiedliche Qualität gibt. Ich glaube nur, dass gerade der Pfarrbrief ein geeignetes Instrument wäre, um Leute zu erreichen, wenn man dieses Instrument entsprechend mit guten Stoffen, gutem Wissen, guten Inhalten aufbauen könnte. Für mich stellt sich die Frage des Kommunikationsmixes so, dass ich glaube, nicht jeder muss alles machen, auch nicht jeder in seinem Bereich alles, sondern es braucht vielleicht eine Kommission oder Arbeitsgruppe, vielleicht gibt es die auch längst, die genau herunterbricht, für welche Zielgruppen eigentlich schon etwas gemacht wird und mit welcher Intention bzw. mit welchem Ergebnis? Sonst haben wir das Problem, dass alle für eine bestimmte Zielgruppe überproportional viel machen, während für neue Ziel- 
gruppen, die uns sehr wichtig sind und unseren Nachwuchs sichern, zu wenig geschieht.

Fischer: Noch ein Satz zum Thema Professionalität: Da müssen wir einfach mehr tun. Wir haben zum Beispiel eigentlich in der katholischen Kirche gar kein Gesicht, mit dem wir in den Medien präsent sein können. $\mathrm{Da}$ ist Kardinal Lehmann vielleicht noch und dann hört es ziemlich schnell auch schon auf. Sendungen wie ,Wort zum Sonntag' und Gottesdienstübertragungen kennen die Leute seit 40 Jahren, die haben ein bestimmtes Profil, zu dem die Leute sagen können: Damit können wir etwas anfangen oder nicht. Bei anderen Sendungen ist das nicht so, das heißt, dass das Profil einfach nicht scharf genug ist oder dass die Kontinuität fehlt. Ein positives Beispiel ist das Kölner Domradio mit sehr viel Musik und einem sehr „unterhaltsamen“ Programm, das man gut nebenbei hören kann. Vielleicht sollten wir in den einzelnen Bereichen sagen: Also eine Sache, wie zum Beispiel einen solchen Kirchensender können wir uns leisten. Oder auch bei den Zeitungen: Warum nicht diese Mitgliederzeitschrift einfach einmal mit ganz neuem Ansatz probieren?

Jooß: Ich bin immer etwas skeptisch bei einem Beispiel, das man dann durch weitere Beispiele erweitern müsste, damit es glaubwürdiger wird. Ich glaube, dass es falsch ist zu sagen: ,Entweder - oder', sondern dass der richtige Weg ein ,Sowohl - als auch' sein muss. Und das heißt für mich, wenn wir über zentrale Strukturen reden, die verbessert werden sollen, müssen wir auch darüber reden, was Nähe und Identität bedeutet. Darum werfe ich auch die Struktur Diözese nicht so einfach weg, sondern sage, wir müssen uns überlegen, wie wir sie wieder lebendig machen können, damit Nähe auch regional erfahren wird. Das ist die eigentliche große Aufgabe und dafür brauchen wir unterschiedliche Medien. Wir brauchen auch eine Inventur im Medienbereich, und da wird man auf die Dauer um die Frage nicht herumkommen, ob die Kirche nicht stärker organisatorisch und finanziell eine Priorität auf die Medien setzen müsste, nicht nur im Rahmen der gestern genannten 17 Millionen. Dabei ist für mich das Ergebnis der Untersuchung wichtig, dass Fernerstehende ihre kirchliche Informations- und Kommunikationsbedürfnisse vor allem aus säkularen Medien befriedigen. Auch hier gilt ein ,Sowohl - als auch': Im Kernbereich müssen wir professionell vertreten sein, aber uns bleibt der Weg nicht erspart, in die säkularen Medien mit allem Selbstbewusstsein hineinzugehen, weil wir damit viele Leute überhaupt erst erreichen. Insofern stört mich ein bisschen diese Mehr-und-Mehr-Regel auf den Kernbereich hin. Ich muss fragen: Wie kann ich säkular die Fernstehenden erreichen? Das ist für mich die zentrale Frage des Überlebens von Kirche in der medialen Öffentlichkeit.

Zwischenfrage aus dem Publikum von Pfarrer Prause: Es geht um Flagge 
zeigen. Die ARD hat vor fünf Jahren eine Untersuchung zur Sendung ,Gott und die Welt' gemacht und uns dann gesagt: Fahren Sie als U-Boot, ändern sie den Titel. Seitdem waren die Einschaltquoten etwas besser. Wir haben im Mitteldeutschen Rundfunk vor einigen Wochen unseren Titel für die Sendung ,Glaubenszeichen' geändert, weil man festgestellt hat, dass er ein Ausschalteffekt war. Das Gleiche haben wir durchgehend in den Sendern, dass wir nicht sagen, wer wir sind, wir sagen auch häufig nicht, was wir wollen. Meine Frage ist, ob wir so viel Wasser in den Wein gießen, dass es allen schmeckt. Wenn ich eine Gaststätte betrete und habe viel Durst, bestelle ich nicht Wein, sondern eine Weinschorle. Aber irgendwann möchte ich ja zum Wein kommen, zum Eigentlichen. Und das ist meine Frage jenseits aller Zahlen, auch auf die Zukunft hin: Wie erkennbar müssen wir sein, und was wirkt?

Theobald: Die Antwort auf diese Frage könnte eine Art Schlusswort von jedem auf dem Podium sein.

Driever: Das Podiumsthema lautet ,Kommunikationsmix', mit dem Untertitel ,Eine Perspektive'. Ich glaube, wir brauchen sehr viele Perspektiven. Und der Kern dieser Perspektiven ist tatsächlich, dass wir authentisch sein müssen, dass wir das Wahre, das Echte nach vorne stellen müssen und nicht unter dem Deckmäntelchen „ein bisschen Religion“. Um diese Perspektiven $z \mathfrak{u}$ erreichen, ist es auch notwendig, ein gutes Stück positiver zu denken. Wir reden viel von unseren Schwächen und zu wenig über unsere Stärken. Wenn wir unsere Perspektiven und unsere Stärken kennen, werden wir letztlich auch draußen bei den Leuten ankommen.

Bergmann: Der Etikettentrick hängt nicht so sehr damit zusammen, dass man den Glauben verstecken muss, sondern dass gegenüber bestimmten Programmen ein gewisses Vorurteil aus 30 Jahren Sehererfahrung herrscht. Solche Sendeformate haben sich überholt, hier muss man neue Zugänge finden und darf diese dann nicht titelmäßig mit den alten Bildern belasten. So gesehen ist die Etikettenänderung vollkommen richtig. Abschliessend noch ein Punkt in eigener Sache über das Wiener Beispiel. Darüber kann ich nur Auskunft geben aus der Phase von 1996 bis 1999 mit einer sehr offensiven Öffentlichkeitsarbeit. Die Mitgliederzeitung gibt es noch, jetzt aber in einem anderen Konzept, in einer sehr defensiven Phase. Das ist das Problem in Wien, dass die Linienfrage nicht geklärt ist. Und das ist ja auch hier die Frage: Tun wir das Richtige? Die Fachleute sind dann dafür da, dass das, was sich als das Richtige erwiesen hat, richtig gemacht wird.

Fischer: Zur Frage nach dem Etikett glaube ich, man darf das eine, also offensive Öffentlichkeitsarbeit zu machen, nicht tun, ohne das andere $z \mathfrak{u}$ lassen, klar und erkennbar Flagge zu zeigen. Und das geht eben über die 
Professionalisierung. Wir sollten unsere Kirchenprogramme und Kirchenzeitungen weiterhin so machen, wie wir sie machen, sie professionalisieren, und das andere nicht lassen. In Erfurt ist in den Adventstagen ein Bischof ins Kaufhaus gegangen und hat sich dort den Leuten gestellt und mit den Leuten über Weihnachten und Advent geredet. Das ist offensiv auf die Leute zugehen. Das muss auch in populären Programmen und Zeitungen vorkommen, daneben muss es natürlich auch noch den großen Bischofsgottesdienst geben ...

Jooß: Was die Sache mit dem Image betrifft: Ich bin gegen Leisetreterei, aber es muss nicht immer der sofortige Einstieg Kirche sein. Es genügt auch, wenn nach einem Kurzbeitrag die Schlussformel kommt: ,Ein Beitrag der katholischen Redaktion.' Da setzt oft ein Aha-Effekt ein, dass man sagt: „Hoppla, das war von der Kirche!“ Und dann ist ein ganz anderer Erkenntnisprozess damit verbunden.

Schulz: Man kann wirklich nicht sagen, was wirkt - dieses oder jenes ist keine Alternative, sondern es muss immer auf die Zielgruppe bezogen sein. Und vor dieser Aufgabe stehen wir jetzt, dass wir die Ergebnisse des Trendmonitors auf Zielgruppen herunterführen müssen. Wir haben gesehen, dass der Kreis der Kirchennahen, der Gläubigen ohnehin medial gut erreicht wird, auch personal, da sind sicher weniger Anstrengungen erforderlich als nach außen zu treten. Ein Trendmonitor kann nur Eckwerte fortschreiben, unter der Oberfläche muss nachgekratzt, müssen Potenziale mit konkreten, konkretisierten, professionell gestalteten Angeboten ausgelotet werden, irgendwie in begrenzten Feldversuchen, um dem Problem der Finanzierung entgegenzukommen. Ich sehe drei Strategien: Einmal die Optimierung der bestehenden Angebote, auch unter Kostengesichtspunkten, auch unter Nutzung von Synergien. Und parallel dazu Erprobung neuer Angebotsformen, wobei wir auf die Erfahrung von ,Echt' oder von ,Dialog“ in Wien durchaus zurückgreifen können, und schliesslich die Strategie, noch stärker als bisher die Kooperation mit säkularen Angeboten zu nutzen. Das sind parallele Dinge und keine Alternativen, vor denen wir stehen, wenn man wirklich alle Chancen nutzen will. 


\section{Ergebnisse der Workshops}

\section{Arbeitsgruppe katholische Presse}

Herausgeber und Journalisten sollten das Erwarten der Leser gleichermaßen als Aufgabe sehen. Orientierung aus eindeutiger katholischer Position einerseits und Dialogforum und Lebenshilfe und Nutzenerweiterung für den Leser andererseits müssen keine Gegensätze sein.

Katholiken als Teil der Gesellschaft spiegeln die Vielfalt des gesellschaftlichen Lebens in Deutschland wider.

Man kann jedoch mit einer Zeitung nicht alle Leser erreichen, aber die „gläubigen kirchennahen“ Katholiken und die „kritischen Kirchenverbundenen" erfordern bei der redaktionellen Positionierung der Zeitung keinen „erheblichen Spagat“.

Die Zeitungen können sich öffnen für „suchende“ Kritische, der treue Kern „bewahrender“ Leser wird diesen Dialog ,aushalten“. Dieser Kern lernt, sich an ein neues Kirchenbild zu gewöhnen. Allerdings „alle zu erreichen“, sollten wir nicht versuchen.

Der publizistische Anspruch katholischer Verleger und Journalisten sollte es sein, mit geeigneten Inhalten und einem professionellen Marketing (Image und Werbung) aus der Gruppe der "kirchlich Distanzierten“ und aus dem so genannten psychologischen Potenzial Leser und Abonnenten für die katholische Presse zu gewinnen.

Die kirchliche Publizistik verliert ihr ureigenstes Terrain an die säkulare Presse. Diese erreicht die der Kirche eng verbundenen und die ihr kritisch verbundenen Katholiken ebenso wie die Glaubensfernen. Hier ist es Aufgabe der katholischen Publizistik, gegenüber den aktuellen Tagesmedien durch orientierende Hintergrundberichterstattung, Glaubenshilfe und Lebenshilfe das bisherige Leserinteresse $z u$ halten und verloren gegangenes Terrain zurück zu gewinnen.

Siegfried Geisbauer, $M D G$

\section{Arbeitsgruppe religiöses Buch}

Der religiöse steht dem allgemeinen Buchmarkt relativ in nichts zurück, was die Anzahl der jährlichen Neuerscheinungen betrifft. $Z u$ viele und zum Teil austauschbare Produkte führen in den Sortimenten und bei den Käufern zu einer Überfrachtung. Gleichzeitig erschwert dies den Zugang zu den allgemeinen Sortimenten, den für den Kauf religiöser Bücher nach wie vor wichtigsten Bezugsweg. 
Das Image religiöser Bücher hat sich im Trendvergleich in Teilbereichen verbessert. Neben Aufmachung und Ausstattung steht und fällt der Erfolg religiöser Literatur am Markt mit der Besetzung und Bearbeitung aktueller Themen und der Glaubwürdigkeit und Kompetenz der Autoren. Es scheint zunehmend schwierig, gute Autorinnen und Autoren zu finden bzw. neue aufzubauen, die die im Trendmonitor enthaltenen Themeninteressen der Leserinnen und Leser in einer verständlichen Sprache vermitteln.

Nach wie vor gibt es bei den Kernzielgruppen religiöser Literatur, beispielsweise bei den Haupt- und Ehrenamtlichen im kirchlichen und caritativen Dienst, ebenso noch $z \mathfrak{u}$ erschließende Potenziale wie im so genannten Öffnungsbereich. Der Katholische Medienverband gibt zu diesem Zweck seinen Mitgliedern mit der Datenbank buchreligion.de ein modernes Marketinginstrument an die Hand. Auf Basis einer umfassenden Verschlagwortung kann so eine Vernetzung der vielfältigen Angebotspalette von Verlagen und religiösen Sortimenten mit den Interessen und Informationsbedürfnissen der Nutzer religiöser Medien gelingen.

Doch werden alle Datenbanken der Welt das umfangreiche religiöse Sortiment vor Ort nicht ersetzen können. Kunden werden sich auch in Zukunft gerne und regelmäßig an den Ort des Einkaufs-Geschehens begeben, falls es in der Nähe eine solche Buchhandlung noch gibt. Sei es, um sich über Neues zu informieren, sich kompetent beraten zu lassen oder einfach auch, weil sie sich dort wohl fühlen. Vorausgesetzt, auch und gerade religiöse Buchhandlungen vermitteln Lust am Lesen und laden zum Schmökern ein.

Religiöse Bücher geben Antworten auf Sinnfragen, helfen den Glauben zu vertiefen und geben neuen Mut. Für Verlage und Buchhandlungen eröffnen sich trotz rückläufiger Tendenzen im allgemeinen Ratgebermarkt interessante Perspektiven zur Erschließung eines wachsenden Markpotenzials. Nun ist es an den Verlegern, die richtigen Bücher zu machen: solche, die sich verkaufen lassen. Und an den Buchhändlern, dass Kunden weiterhin gerne religiöse Bücher und mehr bei ihnen kaufen.

Stefan $E \beta, M D G$

\section{Arbeitsgruppe elektronische Medien}

Potenzial sehen die Teilnehmer in der Zulieferung von Beiträgen zum allgemeinen Programm reichweitenstarker privater Sender. Leider gelingt dies bisher im privaten Fernsehen nicht in dem erforderlichen Maße, im privaten Hörfunk sieht die Situation besser aus. 
Gerade für die Ansprache jüngerer oder kirchenfernerer Katholiken sind neue Formate gefragt. Chancen bieten Unterhaltungssendungen und fiktionale Programme. Eine systematische Erfolgskontrolle sollte die Wirkung solcher Sendungen zeigen.

Gefordert wird auch eine stärkere Bereitschaft von kirchlichen Persönlichkeiten, in den Medien aufzutreten. Um die geforderte Präsenz zu erreichen, sollte eine Sensibilisierung der Bischöfe für die Veränderung in der Gesellschaft stattfinden. Ein systematisches Screening könnte auf regionaler Ebene dazu beitragen, unentdeckte Talente zu finden.

Durch eine solche Personalisierung könnte die Kirche verlorene Glaubwürdigkeit zurückgewinnen. Derzeit können selbst Anfragen der seriösen und reichweitenstarken Talk-Runden nicht mit kirchlichen Persönlichkeiten besetzt werden. Gerade bei Themen, bei denen von „der Kirche" Hoffnung und Hilfe erwartet werden, müssten Würdenträger eine entsprechende Medienpräsenz zeigen. Um zu verhindern, dass dadurch ein einseitiges Bild „der Kirche“ entsteht, dürfen sich Würdenträger nicht auf diese Anlässe beschränken.

Sehr hoch ist der Wiedererkennungswert von Verkündigungssendungen. Deshalb werden diese Formate von Fernseh- und Radiosendern gewünscht. Jedoch erreichen sie oft nur ein kleines und kirchennahes Publikum.

Die Einrichtung eines kirchlichen Spartenkanals stellt sich durch die weitere Digitalisierung und Verspartung im Hörfunk neu. Ziel müsse es sein, den Kirchennahen mit Special Interest Programmen klar profilierte Sendungen zu bieten. Aber gerade dieses Publikum wird von bestehenden Angeboten bereits sehr gut erreicht.

Die knapper werdenden Mittel sollten aber derzeit in reichweitenstarke private Hörfunk- und Fernsehprogramme investiert werden. Bei den öffentlich-rechtlichen Sendern sollten die bestehenden Privilegien verteidigt und es sollte in neue Projekte investiert werden.

Helmut Haimerl und Matthias Veit, MDG

\section{Arbeitsgruppe Öffentlichkeitsarbeit}

Aus den Diskussionsbeiträgen ergab sich, dass die Öffentlichkeitsarbeit/ PR auf diözesaner Ebene Verbesserungspotenziale im Blick auf Zielgruppenkenntnis und ansprache, Qualifikation und Organisation aufweist.

Um die Medienarbeit „auf die Erfordernisse der Mediengesellschaft" auszurichten, müssen Kenntnisse über diverse Zielgruppen und deren Lese- und Informationsverhalten existieren. Marktforschungen, Feldbefragungen, sozio-demographische und nutzertypologische Aspekte sind 
einige Basisgrundlagen, die in vielen Diözesen noch keine ausreichende Würdigung finden.

Voraussetzung für eine zielgruppengerechte Ansprache ist ein strategisches Vorgehen in der Kommunikation. Die Pastoralinstruktion Aetatis Novae spricht von einer Gesamtdarstellung der Kommunikationsstrategien aller kirchlichen Dienste in jedem Bistum. Bei der Formulierung der Strategien findet die Bistumsleitung Unterstützung durch die PRMitarbeiterInnen, denen die operative Umsetzung der Strategien obliegt. Oftmals werden die Öffentlichkeitsarbeiter jedoch in die Entscheidungsfindung der Bistumsleitung zu wenig einbezogen oder sie sind von Gremien ausgeschlossen.

Während sich die journalistische Qualifikation in den Pressestellen in den vergangenen Jahren verbessert hat, besitzen jedoch nur wenige Leiter bischöflicher Pressestellen eine fundierte PR-Ausbildung. Kommunikation wird mitunter als eine Subfunktion der Pressearbeit gesehen.

PR-Referenten und ihre Vorgesetzten sollten einen Umgang des gegenseitigen Vertrauens und der Wertschätzung pflegen. Hierzu gehört es unter anderem, dass sich medienunerfahrene Vorgesetzte, deren Meinung aber von Presse und Rundfunk explizit gewünscht wird, von ihren Mitarbeitern im Umgang mit den Medien (bspw. für das Fernsehen) coachen lassen. Dann kann durch persönliche Ausstrahlung (personal selling) ein positives Image aufgebaut werden, das auch auf andere kirchliche (Medien)Dienste ausstrahlt.

Um eine effektive und effiziente Kommunikation zu betreiben, ist eine Struktur in den Diözesen nötig, die eine koordinierte PR-Arbeit gewährleistet und kostenintensive Doppelarbeiten in diversen Abteilungen (bspw. bei der Konzeption von Plakataktionen, Events oder Mailings) vermeiden hilft.

Adäquate PR-Arbeit ist nicht nur vom finanziellen Budget für Kommunikationsarbeit abhängig: Plakataktionen, wie im Bistum Essen (die auf heiter-ironische Weise auf den Beruf des Geistlichen aufmerksam machen) zeigen, dass mit relativ geringen Mitteln, aber hoher Kreativität, sogar bundesweite positive Resonanz erzielt werden kann.

In ihrer Medien- oder Themenauswahl sind die Diözesen nicht von der Deutschen Bischofskonferenz abhängig. Darin liegt der Vorteil, zeitnah auf Lokales reagieren zu können. Ein großer Nachteil wird jedoch darin gesehen, dass sich die Diözesen untereinander zu wenig für eine gemeinsame Öffentlichkeitsarbeit vernetzen. 